\title{
Expression of NEDD9 in hepatocellular carcinoma and its clinical significance
}

\author{
PENG LU $^{1,2^{*}}$, ZHI-PENG WANG ${ }^{3 *}$, ZHENG DANG $^{4 *}$, ZHI-GANG ZHENG $^{1 *}$, XIAO LI ${ }^{1}$, \\ LIANG ZHOU $^{5}$, RUI DING ${ }^{1}$, SHU-QIANG YUE ${ }^{1}$ and KE-FENG DOU ${ }^{1}$ \\ ${ }^{1}$ Department of Hepatobiliary and Pancreaticosplenic Surgery, Xijing Hospital, The Fourth Military Medical University; \\ ${ }^{2}$ Department of General Surgery, The 518 Central Hospital of PLA; ${ }^{3}$ Department of Pharmacology, School of Pharmacy, \\ The Fourth Military Medical University, Xi'an, Shanxi; ${ }^{4}$ Department of Hepatobiliary Surgery, \\ Lanzhou General Hospital of PLA, Lanzhou, Gansu; ${ }^{5}$ Department of General Surgery, \\ The 155 Central Hospital of PLA, Kaifeng, He'nan, P.R. China
}

Received December 1, 2014; Accepted January 22, 2015

DOI: 10.3892/or.2015.3863

\begin{abstract}
Neural precursor cell expressed, developmentally downregulated 9 (NEDD9) plays an integral role in natural and pathological cell biology. Overexpression of NEDD9 protein has been correlated with poor prognosis in various types of cancer. However, few available data address the precise function of the NEDD9 gene in hepatocellular carcinoma (HCC). In the present study, we investigated NEDD9 expression in 40 primary human HCC tissues compared with matched adjacent non-tumor hepatic tissues using RT-qPCR and western blot analysis. Immunohistochemistry was performed to analyze the correlations between NEDD9 expression and clinicopathological factors. Statistical analyses were applied to derive prognostic values of NEDD9 in HCC. The results showed that the NEDD9 mRNA and protein expression levels in HCC tissues were significantly higher than those in matched adjacent non-tumor hepatic tissues. High NEDD9 expression was correlated with larger tumor size, advanced tumor grade,
\end{abstract}

Correspondence to: Professors Ke-Feng Dou or Shu-Qiang Yue, Department of Hepatobiliary and Pancreaticosplenic Surgery, Xijing Hospital, The Fourth Military Medical University, No. 169 West Changle Road, Xi'an, Shanxi 710032, P.R. China

E-mail: kefeng_dou@126.com

E-mail: shuqiangyue@126.com

"Contributed equally

Abbreviations: HCC, hepatocellular carcinoma; NEDD9, neural precursor cell expressed, developmentally downregulated 9; AFP, $\alpha$-fetoprotein; Cas-L, Crk-associated substrate lymphocyte type; HEF1, human enhancer of filamentation-1; ANHT, adjacent non-tumor hepatic tissues; RT-qPCR, reverse-transcription quantitative polymerase chain reaction; GAPDH, glyceraldehyde-3-phosphate dehydrogenase; RR, relative risk; 95\% CI, 95\% confidence interval

Key words: NEDD9, hepatocellular carcinoma, clinicopathological factors, survival, prognosis metastasis, intrahepatic venous invasion and high UICC TNM stages in HCC patients. Patients with high NEDD9 expression levels exhibited poorer recurrence-free and overall survival than those with a low NEDD9 expression. Additionally, NEDD9 expression status was an independent prognostic factor for survival. This correlation remained significant in patients with early-stage HCC or with normal serum AFP levels. The results of this study suggest that NEDD9 may be a valuable prognostic biomarker for HCC, including early-stage and AFP-normal patients.

\section{Introduction}

Hepatocellular carcinoma (HCC) is the fifth most common malignant tumor due to increases in the rates of viral hepatitis and adiposis hepatica. HCC is also the third leading cause of cancer mortality worldwide $(1,2)$. Each year, $~ 650,000$ HCC cases are reported globally, and according to statistics, China alone accounts for $55 \%$ of HCC cases worldwide $(3,4)$. Hepatic resection is the gold standard therapy for HCC (5). Although the survival of patients with $\mathrm{HCC}$ has been greatly improved owing to advances in surgical improvements and perioperative management, long-term survival after surgical resection remains low due to the high rates of recurrence and metastasis $(6,7)$. The recurrence and metastasis of HCC are multistep processes that involve complex biological and pathological events $(8,9)$. Several molecular pathways and abnormal genetic changes are involved (10)

In daily clinical practice, the prediction of prognosis plays an important role in the assessment of HCC patients and in identifying the optimal therapeutic options. To estimate malignant biological behavior or patient prognosis, sensitive tumor markers are necessary. As a classical and specific marker for HCC, $\alpha$-fetoprotein (AFP) has been widely used for the diagnosis and evaluation of tumor aggressiveness and to determine the prognosis of HCC (11). When a cut-off value of $20 \mathrm{ng} / \mathrm{ml}$ is used, AFP has a sensitivity and specificity for detecting $\mathrm{HCC}$ in the ranges of 41-65 and 80-90\%, respectively (12). However, up to $50 \%$ of patients with HCC have AFP levels $<20 \mathrm{ng} / \mathrm{ml}(13)$, thus AFP cannot be used as a unique target to screen for HCC 
recurrence and to predict patient prognosis. Therefore, it is important to identify useful prognostic biomarkers to predict the risk of this devastating disease and to identify effective therapeutic target molecules that are strongly associated with a poor prognosis in HCC.

Neural precursor cell expressed, developmentally downregulated 9 (NEDD9), also known as Crk-associated substrate lymphocyte type (Cas-L) (14) or human enhancer of filamentation-1 (HEF1) (15), is a focal adhesion scaffold protein that was initially identified by its developmentally regulated expression pattern in the early embryonic, but not adult, mouse brain (16). It also plays an integral role in natural and pathological cell biology (17). Overexpression of NEDD9 protein is correlated with poor prognosis in various types of cancer, including breast cancer (18), glioblastoma (19) and melanoma (20). However, to the best of our knowledge, few available data address the precise function of NEDD9 genes in HCC.

In the present study, we investigated NEDD9 expression in primary human HCC tissues compared with matched adjacent non-tumor hepatic tissues (ANHTs). Immunohistochemistry was performed to analyze the correlation of NEDD9 expression with the clinicopathological factors of patients. Additionally, the potential prognostic utility of NEDD9 expression in HCC was analyzed, including in HCC patients with normal serum AFP levels and with early-stage HCC. The results suggest that NEDD9 may be a valuable prognostic biomarker for HCC.

\section{Materials and methods}

Patients and tissue specimens. In the present study, HCC tissue specimens were obtained from 164 patients with HCC who underwent hepatectomy in the Department of Hepatobiliary and Pancreaticosplenic Surgery, Xijing Hospital (Xi'an, China) from 2004 to 2007. Specimens were obtained from patients who had not received preoperative treatments, such as chemotherapy, ethanol injection, radiofrequency ablation or transarterial chemoembolization. These patients included 133 men and 31 women, and the median patient age was 49 years (range, 29-81 years).

The present study was approved by the Ethics Committee of the Fourth Military Medical University, and it conformed to the ethical guidelines of the 2004 Declaration of Helsinki. Written informed consent was obtained from each patient or from his/her legal guardians. Of the 164 patients with HCC, matched fresh specimens of HCC tissues and ANHTs were collected from 40 random patients, immediately frozen in liquid nitrogen, and subsequently stored at $-80^{\circ} \mathrm{C}$ for reverse-transcription quantitative polymerase chain reaction (RT-qPCR) and western blot analysis. Before the study was initiated, histopathological examinations were performed to confirm that there were sufficient cancer cells in the tumor samples and that no cancer cells had contaminated the ANHTs. All of the specimens were embedded in paraffin and stained with hematoxylin and eosin. The diagnosis in each patient was confirmed by a histopathologist who was blinded to our examination. The data for 10 conventional clinical and pathologic variables were collected for analysis, including gender, age, tumor size, number of tumor nodules, tumor grade, metastasis, venous invasion, UICC TNM stage, HbsAg status and serum AFP level.
RNA extraction, reverse transcription and quantitative PCR. Total RNA was extracted from fresh tissues using TRIzol reagent (Invitrogen, Carlsbad, CA, USA), according to the manufacturer's instructions. cDNA was synthesized from $2 \mu \mathrm{g}$ of RNA using an iScript ${ }^{\mathrm{TM}}$ cDNA Synthesis kit (Bio-Rad, Hercules, CA, USA). Quantitative PCR (qPCR) was performed using a MyiQ2 Two-Color Real-Time PCR Detection System and SYBR-Green dye (both from Bio-Rad), according to the manufacturer's instructions. Oligonucleotide primers were synthesized by Sangon Biotech Co., Ltd. (Shanghai, China). The primers used for the PCR reaction were: NEDD9, forward: 5'-ATG TCC ACG TCT TCC ACC TCC-3' and reverse: 5'-AGT GAC CAG TGC CAT TAG GCT G-3'; and GAPDH, forward: 5'-GAC AGT CAG CCG CAT CTT CT-3' and reverse: 5'-TTA AAA GCA GCC CTG GTG AC-3'. The primer sequences were verified by running a virtual PCR, and the primer concentrations were optimized to avoid primerdimer formation. Additionally, dissociation curves were evaluated to avoid non-specific amplification. The data were analyzed according to the comparative $\mathrm{Ct}$ method and were normalized to GAPDH expression in each sample. All of the experiments were performed in triplicate.

Protein extraction and western blotting. Total protein was extracted using a lysis buffer $(20 \mathrm{mmol} / 1$ Tris- $\mathrm{HCl}, \mathrm{pH} 7.4$; $10 \mathrm{mmol} / 1 \mathrm{NaCl} ; 1 \mathrm{mmol} / 1$ ethylene diamine tetraacetic acid, $\mathrm{pH} 8.0 ; 1 \mathrm{mmol} / 1 \mathrm{MgCl}_{2} ; 1 \% \mathrm{NP}-40 ; 0.1 \%$ sodium dodecyl sulfate; $0.01 \%$ phenylmethylsulfonyl fluoride (Sigma, St. Louis, $\mathrm{MO}, \mathrm{USA}$ ); and $10 \mu \mathrm{l} / \mathrm{ml}$ protease inhibitor cocktail (Promega, Madison, WI, USA) incubated for $20 \mathrm{~min}$ at $4^{\circ} \mathrm{C}$. The protein concentration was determined using the Bio-Rad assay system, and proteins were separated by SDS-polyacrylamide gel electrophoresis and then transferred onto polyvinylidine fluoride membranes (Millipore, Bedford, MA, USA). The blotted membranes were incubated with anti-human NEDD9 antibody (Abcam, Cambridge, UK), followed by a secondary antibody (KPL, Gaithersburg, MD, USA). The $\beta$-actin protein levels were also determined, using this specific antibody (Sigma) as a loading control. Subsequently, the protein bands were detected using the enhanced chemiluminescence detection system (Amersham Pharmacia Biotech, Piscataway, NJ, USA). The western blot analyses were quantified using laser densitometry, and the relative protein expression was then normalized to the $\beta$-actin levels.

Immunohistochemistry and evaluation of staining. Immunohistochemical analysis was performed using the avidin-biotin-peroxidase method for all tissue specimens. Sections were deparaffinized in xylene and dehydrated using a graded alcohol series before endogenous peroxidase activity was blocked with $0.5 \%$ hydrogen peroxide in methanol for $10 \mathrm{~min}$. Non-specific binding was blocked by incubating sections with $10 \%$ normal goat serum in phosphate-buffered saline (PBS) for $1 \mathrm{~h}$ at room temperature. Without washing, the sections were incubated with a monoclonal antibody against human NEDD9 (1:1,000; Abcam; ab18056) in PBS at $4^{\circ} \mathrm{C}$ overnight. Biotinylated goat anti-mouse immunoglobulin G (IgG; 1:400; Abcam; ab128976) was then incubated with the sections for $1 \mathrm{~h}$ at room temperature, and this antibody was detected using streptavidin-peroxidase. Brown color, 
indicative of peroxidase activity, was developed by incubating sections with 0.1\% 3,3'-diaminobenzidine (Sigma; D3939) in PBS with $0.05 \%$ hydrogen peroxide for $5 \mathrm{~min}$ at room temperature. Tissue specimens were assessed separately by two pathologists under double-blind conditions, in which they had no prior knowledge of the clinical or clinicopathological status of the specimens. NEDD9 expression in HCC specimens was evaluated by scanning the entire tissue specimen under low magnification $(\mathrm{x} 40)$ and was confirmed under high magnification (x200 and x400). An immunoreactivity score system was applied as previously described (21). The percentage of positive cells was scored as: $0,<5 \%$, negative; $1,5-25 \%$, sporadic; $2,25-50 \%$, focal; or $3,>50 \%$, diffuse. The staining intensity was scored as: 0 , no; 1 , weak; 2 , moderate; or 3 , strong staining. The NEDD9 immunostaining score was calculated by multiplying the positive cell score by the staining intensity score and thus ranged from 0 to 9 . A high NEDD9 expression was defined as a total score of $\geq 4$, and a low NEDD9 expression level was defined as a total score of $<4$.

Follow-up and prognostic study. Follow-up data were obtained for all the 164 patients. The follow-up period was defined as the interval between the date of surgery and the date of patient death or the last follow up. Death from other causes was treated as censored events. Recurrence was diagnosed by ultrasonography or computed tomography scans. Recurrence-free survival was defined as the length of time after hepatectomy during which a patient survived without signs of HCC.

Statistical analysis. Statistical analysis was performed using SPSS software, version 17.0 (IBM SPSS, Chicago, IL, USA). Each experiment was repeated at least three times, and all data are summarized and presented as the mean \pm SD. The differences between means were statistically analyzed using the t-test. The $\chi^{2}$ test for proportions was used to analyze the relationships between NEDD9 expression and various clinicopathological factors. Survival curves were calculated using the Kaplan-Meier method and were compared using the log-rank test. Cox proportional hazard analysis was used to explore the effects of clinicopathological factors and NEDD9 expression on survival. SPSS statistical software was used to perform the univariate analysis of each variance and identify the factors that were significantly different in the two groups, followed by a multivariate analysis. $\mathrm{P}$-values were two-sided, and $\mathrm{P}<0.05$ was considered to indicate a statistically significant result.

\section{Results}

Analysis of NEDD9 $m R N A$ expression. mRNA expression levels of NEDD9 were evaluated in 40 pairs of resected specimens (consisting of tumor tissue samples and matched ANHT samples) from patients with HCC using RT-qPCR. We found that 27 of the 40 patients $(67.5 \%)$ had higher NEDD9 mRNA expression levels in HCC tissues than in ANHTs. The results indicated that the relative expression levels of NEDD9 mRNA in HCC tissues were significantly higher than those in ANHTs (4.38 \pm 0.65 vs. $3.21 \pm 0.71, \mathrm{P}<0.01$; Fig. 1 ).

Analysis of NEDD9 protein expression. NEDD9 protein levels in resected HCC tissues and ANHT samples were measured

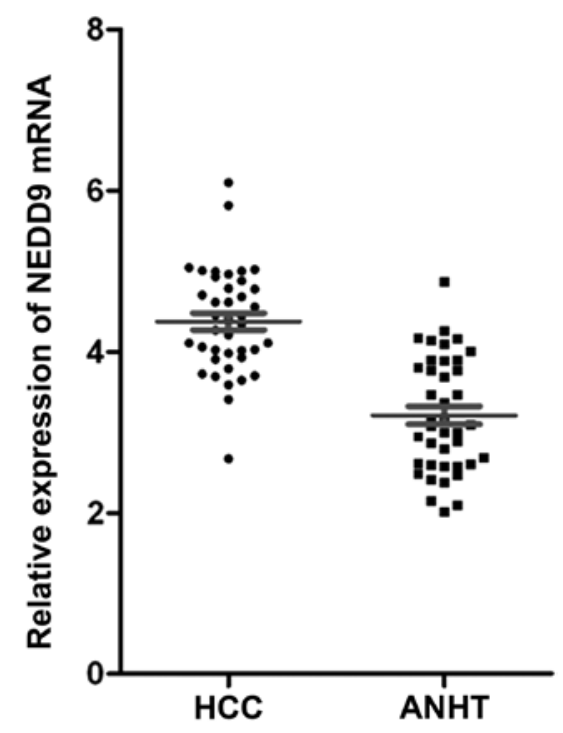

Figure 1. RT-qPCR analysis of NEDD9 expression in HCC patients. The NEDD9 mRNA levels were higher in HCC tissues than in ANHTs $(\mathrm{P}<0.01)$. NEDD9, neural precursor cell expressed, developmentally downregulated 9; HCC, hepatocellular carcinoma; ANHTs, adjacent non-tumor hepatic tissues.

by western blot analysis. NEDD9 expression was increased in 26 of the 40 tumor tissue samples (65\%), compared with the matched ANHT samples. The relative expression levels of NEDD9 protein in HCC tissues were significantly higher than in matched ANHT samples $(1.24 \pm 0.45$ vs. $0.68 \pm 0.19, \mathrm{P}<0.01$; Fig. 2).

NEDD9 immunohistochemistry. NEDD9 expression was mainly localized within the cytoplasm. NEDD9 immunoreactivity varied between HCC tissue samples and matched ANHT samples. There was no significant NEDD9 expression in ANHTs or only weak staining in the cytoplasm. As shown in Fig. 3, the NEDD9 expression patterns differed among HCC tissues. NEDD9 staining was negative (score of 0) in $19 \mathrm{HCC}$ samples, whereas weak positive staining (score of $>0$ and $<4$ ) was detected in $61 \mathrm{HCC}$ samples, moderate positive staining (score of $\geq 4$ and $<6$ ) was detected in 49 HCC samples, and strong positive staining (score of $\geq 6$ ) was detected in $35 \mathrm{HCC}$ samples.

Relationship between NEDD9 expression and clinicopathological characteristics. According to the immunohistochemical results (Fig. 3), the HCC tissues were divided into two groups based on the NEDD9 expression: the low expression group (negative + weak, $n=80$ ) and the high expression group (moderate + strong, $n=84$ ). The correlations between NEDD9 expression and HCC patient clinicopathological characteristics were analyzed (Table I). We demonstrated that tumor size was larger $(\mathrm{P}=0.017)$, tumor grade was more advanced $(\mathrm{P}=0.021)$, metastasis and intrahepatic venous invasion were more frequent $(\mathrm{P}=0.003$ and $\mathrm{P}<0.001)$, and UICC TNM stage was higher $(\mathrm{P}=0.034)$ in the high expression group than in the low expression group. However, there were no significant differences of genders $(\mathrm{P}=0.726)$, ages $(\mathrm{P}=0.831)$, numbers of tumor nodules $(\mathrm{P}=0.312)$, HbsAg status $(\mathrm{P}=0.672)$ or AFP level $(\mathrm{P}=0.336)$ between the high and low expression groups. 


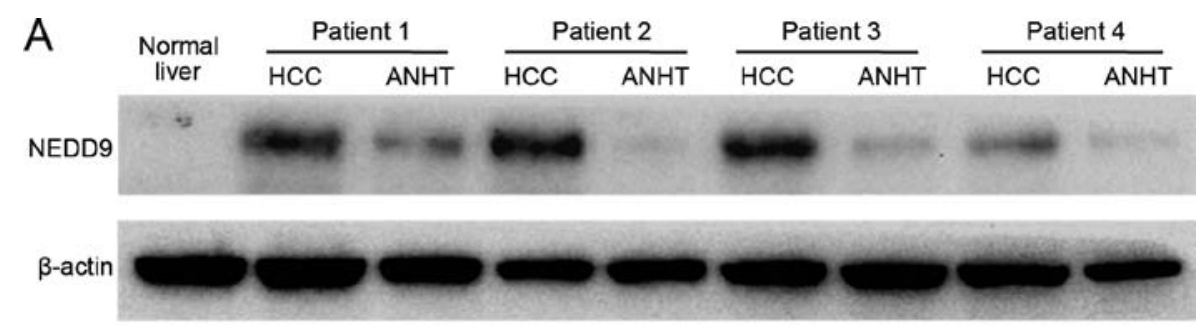

B

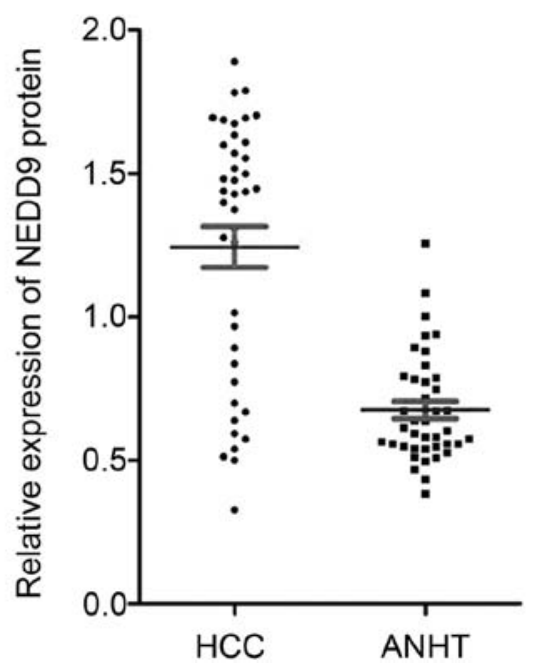

Figure 2. Western blot analysis of NEDD9 expression in HCC patients. The NEDD9 protein levels were higher in HCC tissues than in ANHTs (P<0.01). NEDD9, neural precursor cell expressed, developmentally downregulated 9; HCC, hepatocellular carcinoma; ANHTs, adjacent non-tumor hepatic tissues.
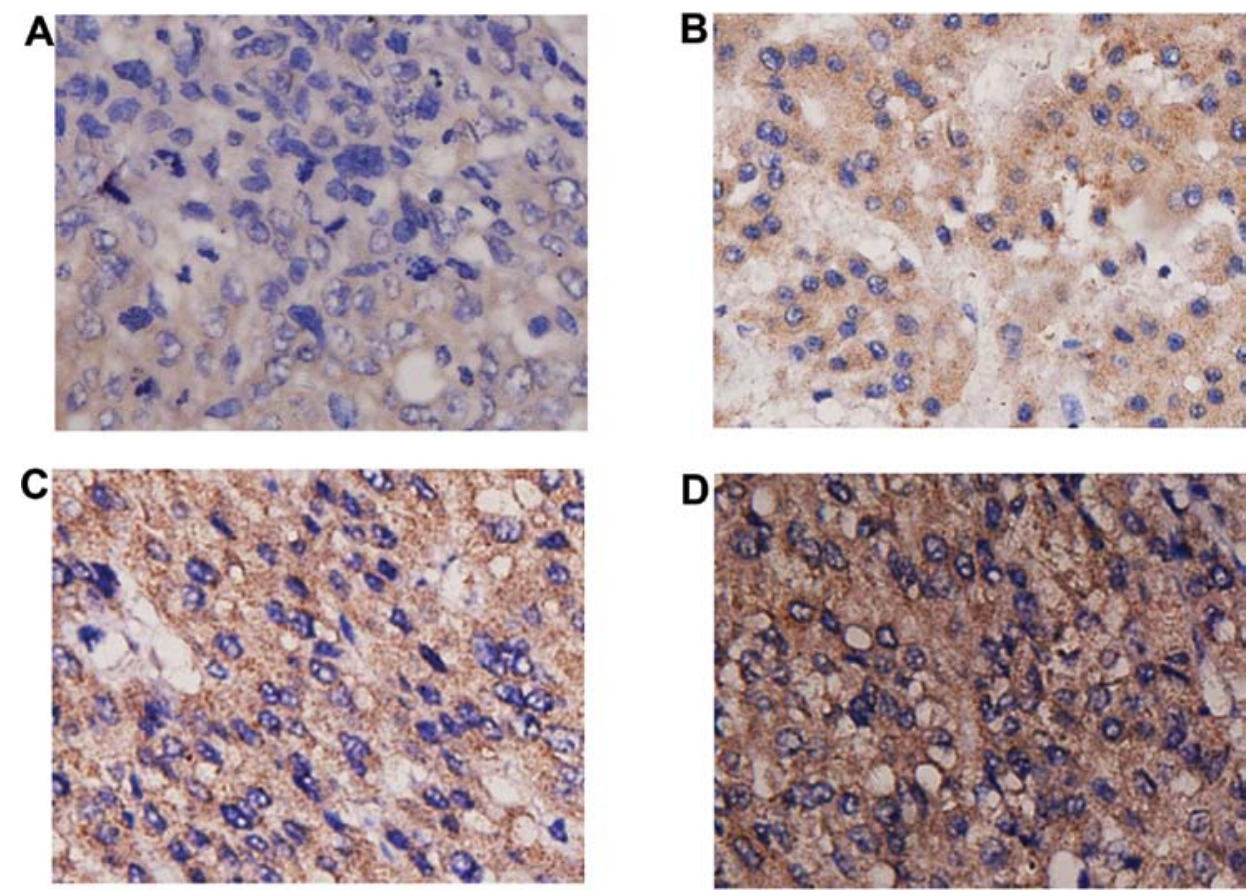

Figure 3. Different NEDD9 expression levels in HCC tissues: (A) negative, (B) weak, (C) moderate and (D) strong. NEDD9, neural precursor cell expressed, developmentally downregulated 9; HCC, hepatocellular carcinoma.

These results indicated that NEDD9 may be involved in the differentiation, metastasis, invasion and progression of HCC.

Correlations of NEDD9 expression with the recurrence and prognosis of HCC. Since the level of NEDD9 expression was correlated with tumor size, tumor grade, metastasis, intrahepatic venous invasion and UICC TNM stage, we further hypothesized that the level of NEDD9 expression would influence the recurrence and prognosis of patients suffering from HCC. Subsequently, post-operative Kaplan-Meier 
Table I. Correlations between NEDD9 expression and clinicopathological characteristics in 164 patients with hepatocellular carcinoma.

\begin{tabular}{|c|c|c|c|c|}
\hline \multirow{2}{*}{$\begin{array}{l}\text { Clinicopathological } \\
\text { characteristics }\end{array}$} & \multirow[b]{2}{*}{$\mathrm{n}$} & \multicolumn{2}{|c|}{$\begin{array}{c}\text { NEDD9 } \\
\text { expression levels }\end{array}$} & \multirow[b]{2}{*}{ P-value } \\
\hline & & Low & High & \\
\hline Gender & & & & 0.726 \\
\hline Male & 133 & 64 & 69 & \\
\hline Female & 31 & 16 & 15 & \\
\hline Age (years) & & & & 0.831 \\
\hline$\leq 55$ & 112 & 54 & 58 & \\
\hline$>55$ & 52 & 26 & 26 & \\
\hline Tumor size $(\mathrm{cm})$ & & & & $0.017^{\mathrm{a}}$ \\
\hline$\leq 5$ & 89 & 51 & 38 & \\
\hline$>5$ & 75 & 29 & 46 & \\
\hline No. of tumor nodules & & & & 0.312 \\
\hline Solitary & 117 & 60 & 57 & \\
\hline Multiple ( $\geq 2$ ) & 47 & 20 & 27 & \\
\hline $\begin{array}{l}\text { Tumor grade } \\
\text { (differentiation) }\end{array}$ & & & & $0.021^{\mathrm{a}}$ \\
\hline Well & 44 & 28 & 16 & \\
\hline Moderate or poor & 120 & 52 & 68 & \\
\hline Metastasis & & & & $0.003^{\mathrm{a}}$ \\
\hline Absence & 120 & 67 & 53 & \\
\hline Presence & 44 & 13 & 31 & \\
\hline Venous invasion & & & & $<0.001^{\mathrm{a}}$ \\
\hline Absence & 112 & 69 & 43 & \\
\hline Presence & 52 & 11 & 41 & \\
\hline UICC TNM stage & & & & $0.034^{\mathrm{a}}$ \\
\hline $\mathrm{I}+\mathrm{II}$ & 97 & 54 & 43 & \\
\hline $\mathrm{III}+\mathrm{IV}$ & 67 & 26 & 41 & \\
\hline HBsAg & & & & 0.672 \\
\hline Positive & 148 & 73 & 75 & \\
\hline Negative & 16 & 7 & 9 & \\
\hline Serum AFP (ng/ml) & & & & 0.336 \\
\hline$\geq 20$ & 88 & 46 & 42 & \\
\hline$<20$ & 76 & 34 & 42 & \\
\hline
\end{tabular}

a Significant P-value. NEDD9, neural precursor cell expressed, developmentally downregulated 9; AFP, $\alpha$-fetoprotein.

survival curves were used to evaluate the recurrence-free survival and overall survival rates of patients with HCC relative with their levels of NEDD9 expression. The log-rank test showed that both recurrence-free and overall survival rates were significantly different between low and high NEDD9 expression groups $(\mathrm{P}<0.01$ each). The low NEDD9 expression group demonstrated increased recurrence-free and overall survival rates compared with the high NEDD9 expression group (Fig. 4). The cumulative 5-year recurrence-free survival rate was $2.4 \%$ in the high NEDD9 expression group but $16.3 \%$
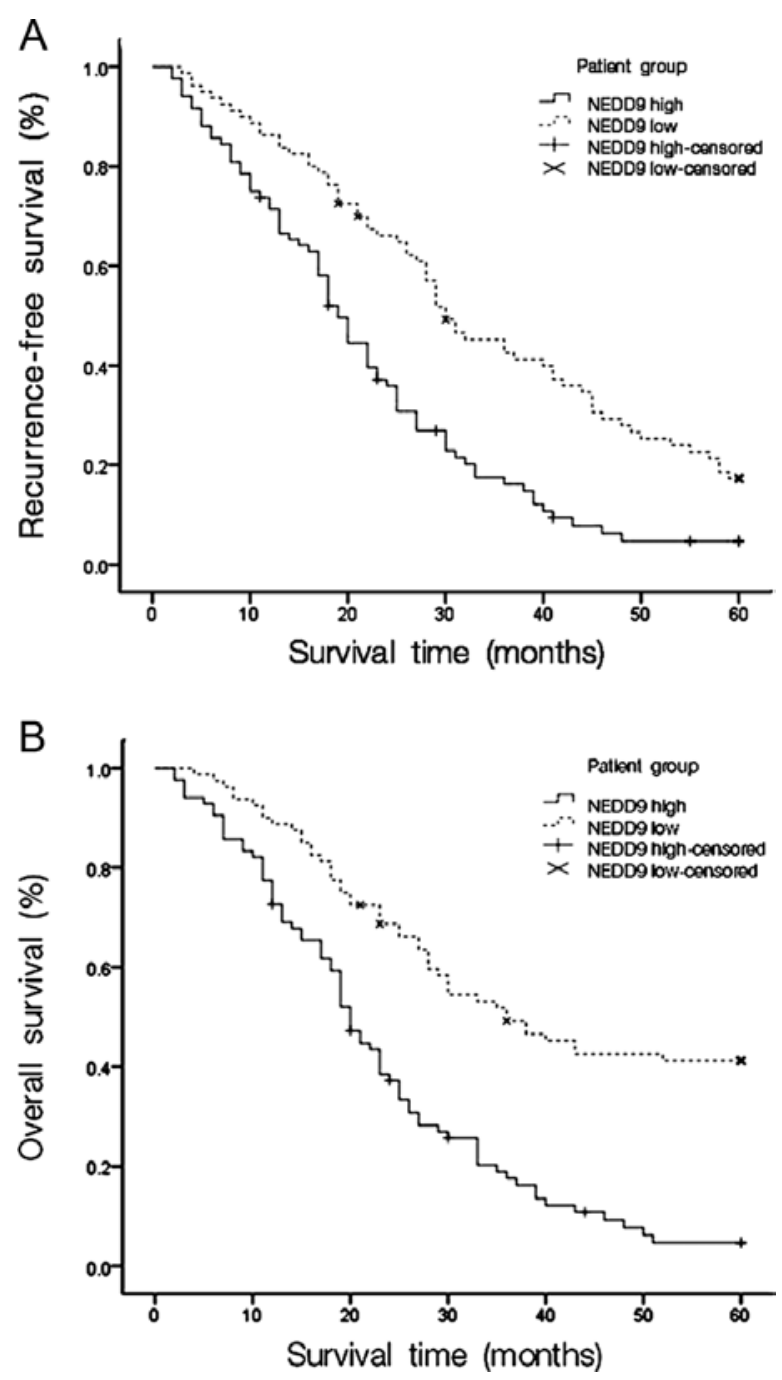

Figure 4. (A) Recurrence-free and (B) overall survival curves were constructed using the Kaplan-Meier method and were evaluated using the log-rank test $(\mathrm{P}<0.01$ each).

in the low NEDD9 expression group. The cumulative 5-year overall survival rate was $3.6 \%$ in the high NEDD9 expression group but $38.8 \%$ in the low NEDD9 expression group.

Univariate and multivariate analyses. Univariate Cox regression analysis showed that tumor size, metastasis, venous invasion, UICC TNM stage and NEDD9 protein expression levels were significantly associated with HCC patient overall survival. Furthermore, to evaluate the potential role of high NEDD9 expression levels as an independent predictor of overall survival among HCC patients, multivariate Cox regression analyses were performed. The results indicated that only metastasis, venous invasion, UICC TNM stage and NEDD9 expression independently predicted overall survival among HCC patients (Table II).

Prognostic values of NEDD9 in different HCC subgroups. To demonstrate the value of NEDD9 expression in predicting the survival of HCC patients, multiple analysis methods were performed in the present study. We proved that the recurrencefree and overall survival times of patients expressing low levels of NEDD9 in their HCC lesions were much longer than those 
Table II. Univariate and multivariate Cox regression analysis for the overall survival of 164 patients with HCC.

\begin{tabular}{|c|c|c|c|c|c|c|}
\hline \multirow{2}{*}{$\begin{array}{l}\text { Clinicopathological } \\
\text { characteristics }\end{array}$} & \multirow[b]{2}{*}{$\mathrm{RR}$} & \multicolumn{2}{|c|}{ Univariate analyses } & \multicolumn{2}{|c|}{ Multivariate analyses } & \multirow[b]{2}{*}{ P-value } \\
\hline & & $95 \% \mathrm{CI}$ & P-value & RR & $95 \% \mathrm{CI}$ & \\
\hline Gender & 1.193 & $0.769-1.852$ & 0.431 & 0.751 & $0.453-1.243$ & 0.265 \\
\hline Age (years) & 0.885 & $0.599-1.305$ & 0.537 & 1.085 & $0.703-1.675$ & 0.713 \\
\hline Tumor size & 1.552 & $1.085-2.220$ & $0.016^{\mathrm{a}}$ & 1.109 & $0.756-1.627$ & 0.579 \\
\hline No. of tumor nodules & 1.082 & $0.733-1.597$ & 0.692 & 0.688 & $0.424-1.115$ & 0.129 \\
\hline Tumor grade & 1.057 & $0.709-1.576$ & 0.786 & 0.784 & $0.517-1.186$ & 0.249 \\
\hline Metastasis & 2.174 & $1.482-3.190$ & $<0.01^{\mathrm{a}}$ & 0.917 & $1.171-3.139$ & $0.01^{\mathrm{a}}$ \\
\hline Venous invasion & 3.423 & $2.346-4.995$ & $<0.01^{\mathrm{a}}$ & 2.735 & $1.750-4.274$ & $<0.01^{\mathrm{a}}$ \\
\hline UICC TNM stage & 2.040 & $1.423-2.923$ & $<0.01^{\mathrm{a}}$ & 1.806 & $1.222-2.670$ & $<0.01^{\mathrm{a}}$ \\
\hline HBsAg & 1.144 & $0.644-2.035$ & 0.646 & 1.278 & $0.641-2.547$ & 0.485 \\
\hline Serum AFP & 1.260 & $0.883-1.798$ & 0.203 & 1.032 & $0.704-1.513$ & 0.873 \\
\hline NEDD9 level & 0.365 & $0.251-0.532$ & $<0.01^{\mathrm{a}}$ & 1.618 & $1.050-2.493$ & $0.029^{\mathrm{a}}$ \\
\hline
\end{tabular}

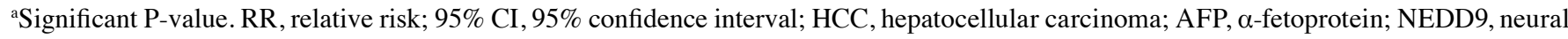
precursor cell expressed, developmentally downregulated 9.

A

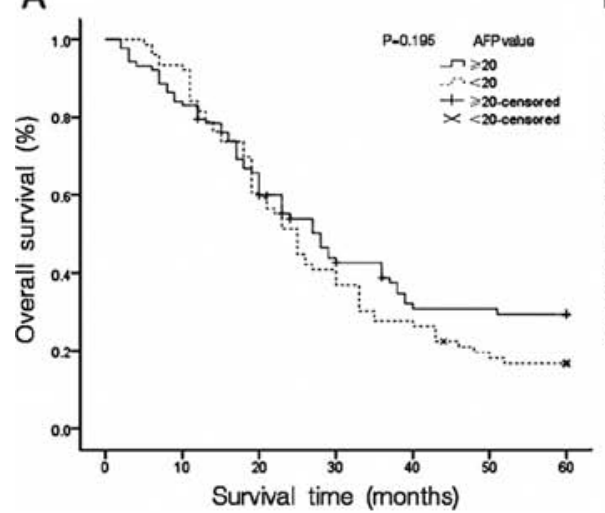

$\mathrm{D}$

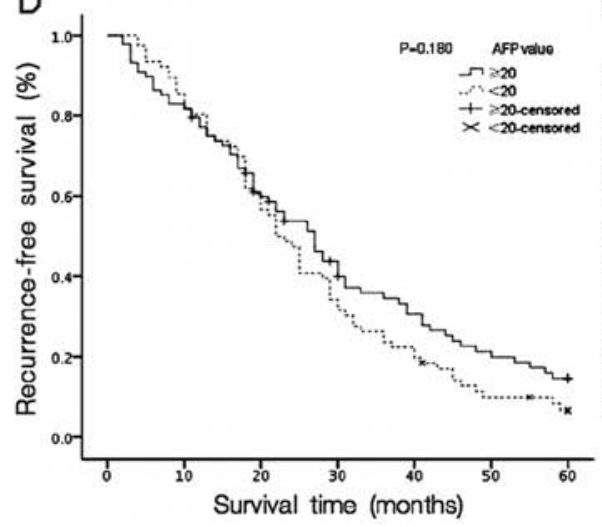

B

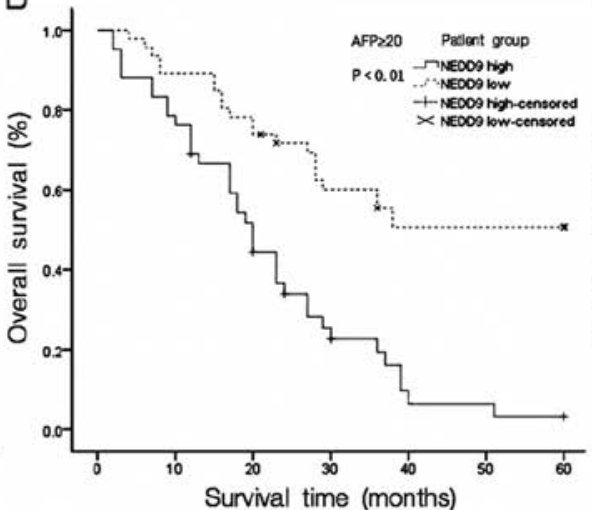

E

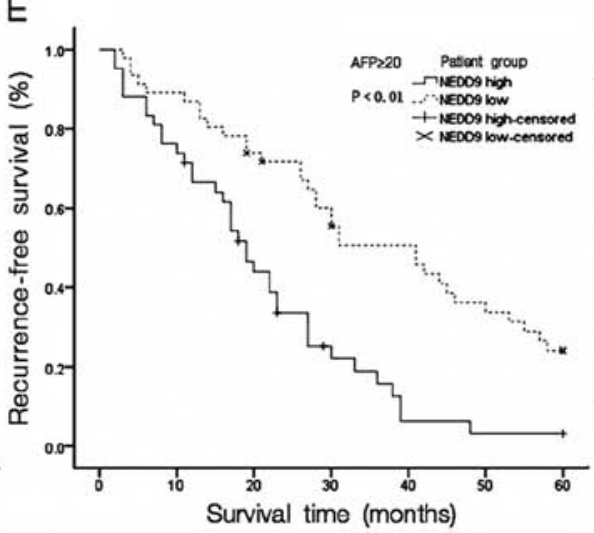

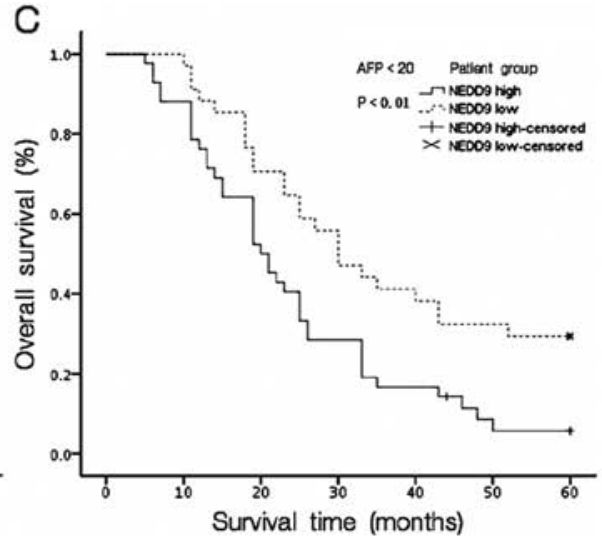

F

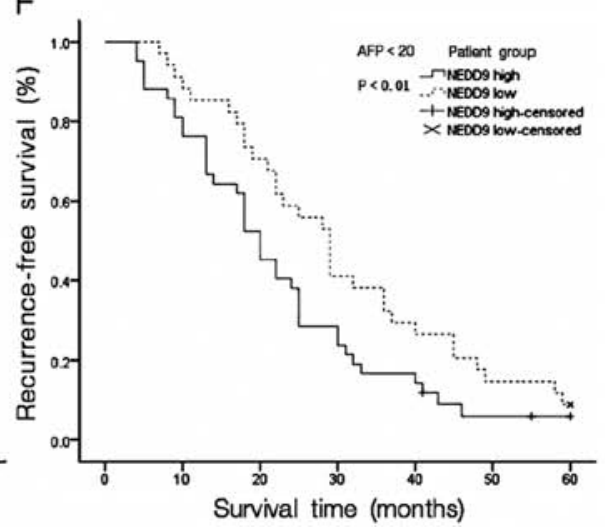

Figure 5. Kaplan-Meier analysis of overall and recurrence-free survival in 164 patients, based on NEDD9 expressions in HCC subgroups with different AFP levels. Using AFP level $(20 \mathrm{ng} / \mathrm{ml})$ as the cut-off could not separate patients with different overall $(\mathrm{A}, \mathrm{P}=0.195)$ and recurrence-free $(\mathrm{D}, \mathrm{P}=0.180)$ rates in the study cohort. In contrast, the NEDD9 expression level predicted different overall survival rates in the subgroups with AFP $\geq 20$ ng/ml (B, P $\leq 0.01)$ and AFP $<20 \mathrm{ng} / \mathrm{ml}(\mathrm{C}, \mathrm{P} \leq 0.01)$. The NEDD9 expression level also predicted different recurrence-free survival rates of patients with $\mathrm{AFP} \geq 20 \mathrm{ng} / \mathrm{ml}(\mathrm{E}, \mathrm{P} \leq 0.01)$ and AFP $<20$ ng/ml (F, P $\leq 0.01)$. NEDD9, neural precursor cell expressed, developmentally downregulated 9; HCC, hepatocellular carcinoma; AFP, $\alpha$-fetoprotein.

with high NEDD9 expression (Fig. 4). Notably, these patients with different recurrence-free and overall survival could not be distinguished by conventional AFP testing. In other words, we could not use negative serum AFP to predict HCC patients' recurrence and prognosis. A validation cohort was employed to evaluate further the prognostic value of NEDD9 for specific subgroups of patients. We used a cut-off level of $20 \mathrm{ng} / \mathrm{ml}$ AFP to subgroup the 164 HCC patients, and we evaluated the 
A

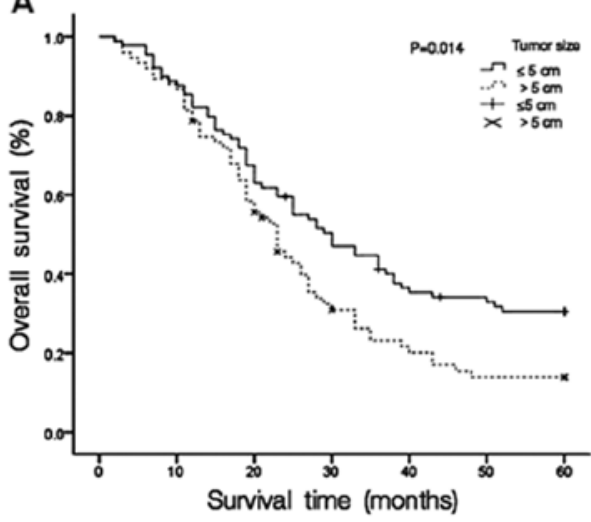

D

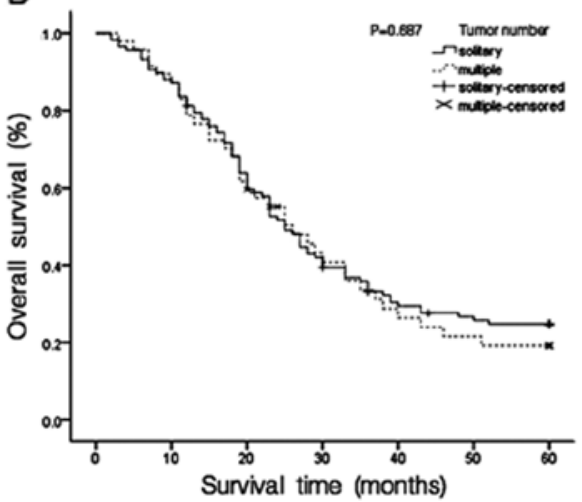

G

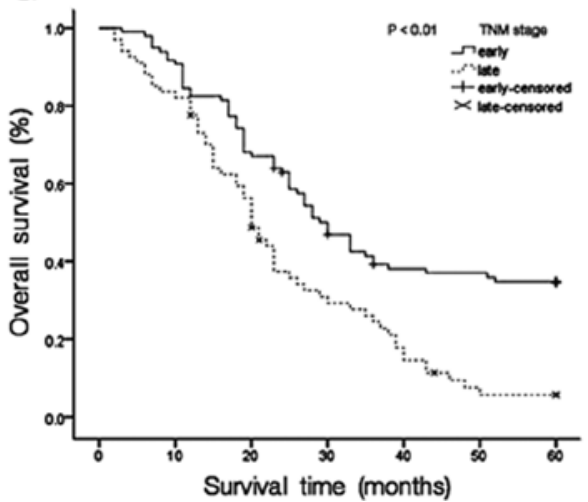

B

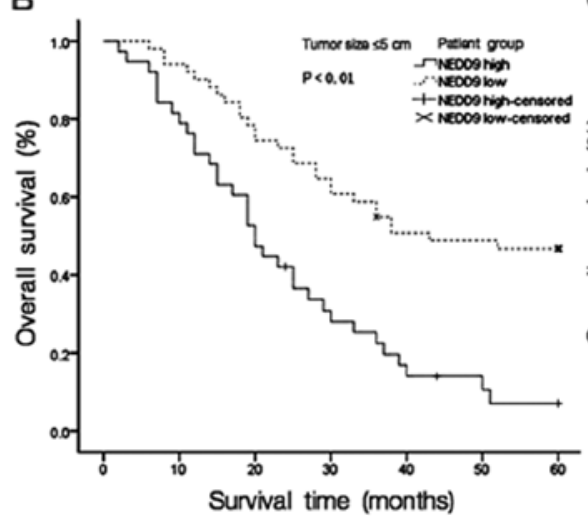

E

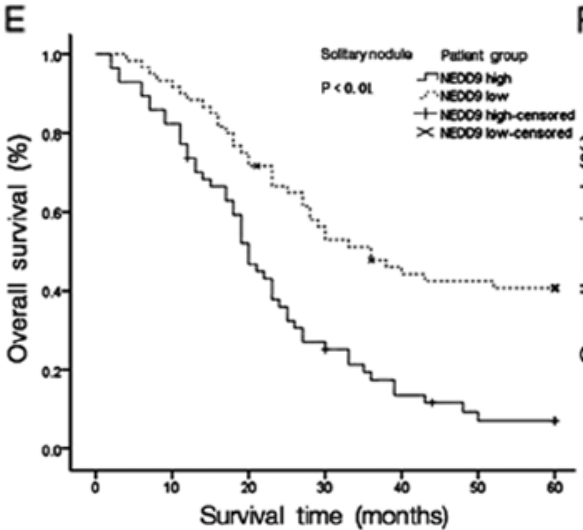

$\mathrm{H}$

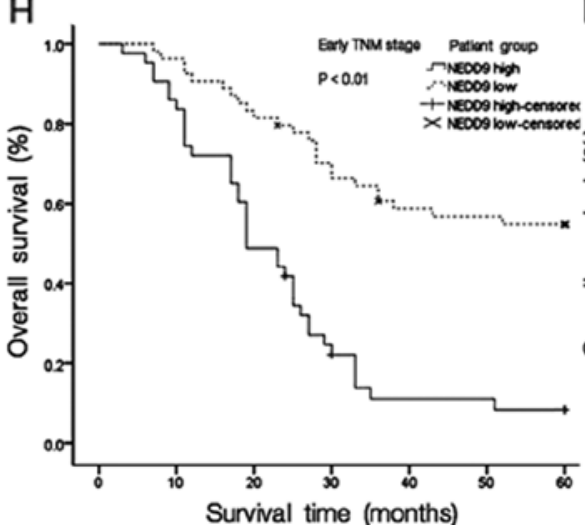

C

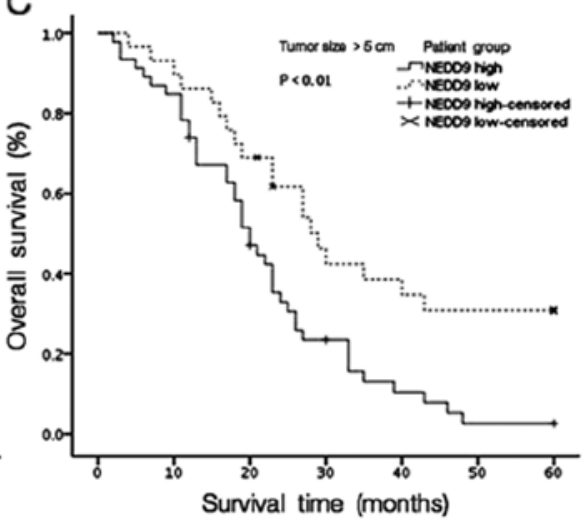

$\mathrm{F}$
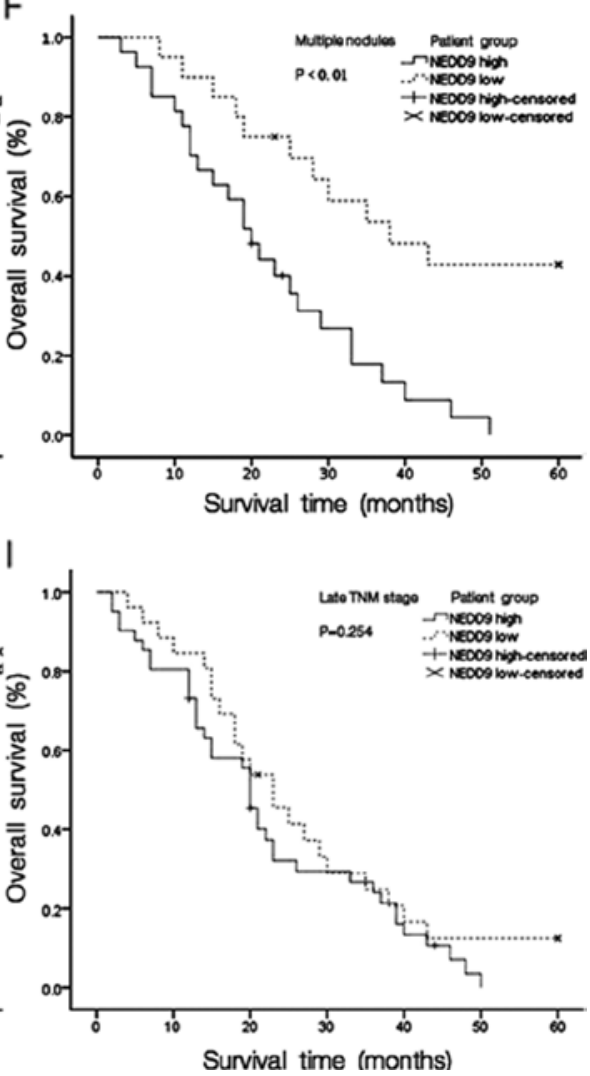

Figure 6. Kaplan-Meier analysis of overall survival in 164 patients, based on NEDD9 expression in the HCC clinical subgroups. (A) Tumor size and (G) TNM stage, but not the number of (D) tumor nodules, distinguished patients with different overall survival rates in the study cohort. (B and C) When patients were divided into subclinical groups according to tumor size, the probabilities of survival with either HCC lesion diameter $>5$ or $\leq 5 \mathrm{~cm}$ and high and low NEDD9 expression, distinguished lower and higher overall survival rates, respectively. (E and F) Overall survival in patients with single tumor lesions and multi-tumor lesions. (H and I) Overall survival rates in patients classified into early TNM stages (I+II) and late TNM stages (III+IV), as differentiated by a high or low NEDD9 expression. NEDD9, neural precursor cell expressed, developmentally downregulated 9; HCC, hepatocellular carcinoma.

prognostic significance of NEDD9 in the patient subgroups. Our data suggested that the AFP cut-off value of $20 \mathrm{ng} / \mathrm{ml}$ was not significantly predictive of patient survival $(\mathrm{P}=0.195)$, whereas the NEDD9 levels were significantly associated with overall survival in patients with serum AFP that was either positive ( $\geq 20 \mathrm{ng} / \mathrm{ml}$ ) or negative $(<20 \mathrm{ng} / \mathrm{ml})(\mathrm{P}<0.01)$ (Fig. 5).

In the clinic, it is difficult to predict survival in $\mathrm{HCC}$ patients with tumors $<5 \mathrm{~cm}$ in diameter, single tumor nodules and early tumor stages (UICC TNM stages I-II), and in these early-stage HCC subgroups of our entire study cohort, NEDD9 expression level presented efficient predictive power for predicting patient prognosis. In the subgroup with tumor size $<5 \mathrm{~cm}$ in diameter, the 5-year overall survival rate was $45.1 \%$ in the low NEDD9 group, compared with $5.26 \%$ for patients exhibiting a high NEDD9 expression ( $\mathrm{P}<0.01$, Fig. $6 \mathrm{~B})$. In the clinical subgroup with single neoplastic nodules, low NEDD9 expression patients revealed a 5-year survival rate of $38.3 \%$, whereas the overall survival rate decreased to $5.26 \%$ in the high NEDD9 group ( $\mathrm{P}<0.01$, Fig. 6E). In early TNM stage HCC patients (TNM stages I-II), the 5-year overall survival rates were 51.9 and $6.98 \%$, respectively, for low and high NEDD9 expression patients $(\mathrm{P}<0.01$, Fig. $6 \mathrm{H})$. Collectively, our results indicate that, in various HCC clinical subgroups or even with difficult-to-predict survival, NEDD9 expression is a promising prognostic factor. 


\section{Discussion}

NEDD9 is one of a four-member family of protein scaffold and adaptor molecules, and it plays a central role in transducing signals from cell surface receptors and cytoplasmic protein tyrosine kinases (PTKs). NEDD9 has no catalytic activity but instead exerts its influence on cell behavior by functioning as a platform for the assembly of multiprotein complexes (22). The expression of NEDD9 is tissue-specific, and high levels of NEDD9 mRNA and protein expression are observed in the lung, kidney, fetal brain and tissues, which are rich in immature lymphoid cells (15). NEDD9 is abundant in tumor cell lines derived from epithelial lineages, in glioblastomas (19) and in lymphoma cell lines (23). Findings of previous studies have shown that NEDD9 overexpression, as caused by oncogenic signaling abnormalities, is associated with metastasis in different types of carcinomas $(19,20,25,26)$ and has been associated with drug resistance in gastrointestinal stromal tumors (27). However, to the best of our knowledge, few available data address the involvement of the NEDD 9 gene in HCC.

In the present study, we first showed that the expression of NEDD9 mRNA and protein in HCC tissues was significantly higher than that in matched ANHTs. Additionally, high NEDD9 expression was correlated with larger tumor size, advanced tumor grade, metastasis, intrahepatic venous invasion and high UICC TNM stages in HCC patients. Survival analyses indicated that patients with a high NEDD9 expression showed poorer recurrence-free and overall survival than those with a low NEDD9 expression, and the status of NEDD9 expression was an independent prognostic factor.

AFP is most commonly employed in clinics for HCC screening worldwide and as an important predictor of survival and tumor recurrence following tumor resection among serum AFP-positive HCC patients (28-30). When used independently, the diagnostic sensitivity of AFP for HCC is not satisfactory, leading to a large number of HCC patients without AFP elevation not being diagnosed and subsequently progressing to late-stage HCC, when they become clinically symptomatic and detectable (29). In the present study cohort, only $53.7 \%$ of HCC patients were AFP-positive. Due to its poor sensitivity in screening for new HCC cases that have not been detected by imaging technology previously, AFP is only unsatisfactorily effective in specific patient populations (29). By comparison, in the patient group in which AFP levels did not predict prognosis or cancer recurrence, NEDD9 appeared to be a powerful indicator of overall and recurrence-free survival times among different patients. Thus, the present study has demonstrated the potential value of NEDD9 in predicting patient survival and recurrence in subgroups with normal AFP levels, which may have proven difficult using the current clinically available biomarkers.

The classification of HCC by anatomic disease extent, such as UICC TNM stage, is commonly used in clinics to determine the appropriate treatment and prognosis. It is an increasingly important component of cancer surveillance and control and an endpoint for the evaluation of populationbased screening and early detection efforts. Small tumor size $(\leq 5 \mathrm{~cm})$, single tumor nodules, and other factors contribute to the early staging of HCC, most cases of which can be effectively treated by strategies including surgical anatomic hepatectomy or liver transplantation. Nevertheless, some HCC patients have the potential to progress to poor survival even though they have employed curative treatment. In our validation cohort, early-stage HCC patients (tumor size $\leq 5 \mathrm{~cm}$ and TNM stage I-II) showed relatively higher overall survival rates than late-stage HCC patients (tumor size $\geq 5 \mathrm{~cm}$ and TNM stage III-IV) $(\mathrm{P}<0.01$ each). Notably, early-stage HCC patients (tumor size $\leq 5 \mathrm{~cm}$, single tumor nodules and TNM stage I-II) expressing low levels of NEDD9 exhibited significantly improved overall survival than those expressing high levels of NEDD9, which was consistent with our observations in late-stage HCC patients (tumor size $>5 \mathrm{~cm}$, multiple tumor nodules and TNM stage III-IV).

Using biomarkers to identify patients who are at higher risk of developing worse prognosis may reduce mortality and medical costs. In conclusion, to the best of our knowledge, we have demonstrated for the first time that the NEDD9 expression level was strongly associated with the prognoses of HCC patients. The available evaluation systems for HCC remain limited in providing predictive information for determining therapeutic strategies in subgroups of HCC patients, such as predicting the prognosis of early-stage HCC and AFP-negative HCC. The present findings provide new evidence that a higher expression of NEDD9 in HCC may be important for detecting an aggressive phenotype or a phenotype predicting poor prognosis. The present study indicates that NEDD9 serves as a diagnostic biomarker for HCC that may improve prospects for the early detection of HCC and that an improved rate of detection may have important prognostic implications for patients with HCC. Of note, the current study was of a retrospective nature, and the number of patients was small. Clearly, further prospective studies designed to include a larger number of HCC lesions are needed to validate the conclusions of the present study. Moreover, it would be of great clinical value to determine the relationships of NEDD9 with other signaling molecules and pathways, which may be useful in obtaining a better understanding of the molecular pathogenesis of these tumors and to develop more effective targeted therapeutic strategies.

\section{Acknowledgements}

This study was edited for proper English language, grammar, punctuation, spelling and overall style by one or more of the highly qualified native English speaking editors at American Journal Experts. This study was supported by grants from the National Natural Science Foundation of China (81172291 and 81101818).

\section{References}

1. Nordenstedt H, White DL and El-Serag HB: The changing pattern of epidemiology in hepatocellular carcinoma. Dig Liver Dis 42 (Suppl 3): S206-S214, 2010.

2. Parkin DM, Bray F, Ferlay J and Pisani P: Global cancer statistics, 2002. CA Cancer J Clin 55: 74-108, 2005.

3. Asia-Pacific Working Party on Prevention of Hepatocellular Carcinoma: Prevention of hepatocellular carcinoma in the AsiaPacific region: consensus statements. J Gastroenterol Hepatol 25: 657-663, 2010.

4. Yuen MF, Hou JL, Chutaputti A; Asia Pacific Working Party on Prevention of Hepatocellular Carcinoma: Hepatocellular carcinoma in the Asia pacific region. J Gastroenterol Hepatol 24: 346-353, 2009. 
5. Schwartz ME and Shrager B: Surgical resection for hepatocellular carcinoma in the noncirrhotic: the Western experience. Recent Results Cancer Res 190: 85-100, 2013.

6. Yang Y, Nagano H, Ota H, et al: Patterns and clinicopathologic features of extrahepatic recurrence of hepatocellular carcinoma after curative resection. Surgery 141: 196-202, 2007.

7. Tang ZY, Ye SL, Liu YK, et al: A decade's studies on metastasis of hepatocellular carcinoma. J Cancer Res Clin Oncol 130 187-196, 2004

8. Yang LY, Tao YM, Ou DP, Wang W, Chang ZG and Wu F: Increased expression of Wiskott-Aldrich syndrome protein family verprolin-homologous protein 2 correlated with poor prognosis of hepatocellular carcinoma. Clin Cancer Res 12 : 5673-5679, 2006.

9. Okuda K: Hepatocellular carcinoma: clinicopathological aspects. J Gastroenterol Hepatol 12: S314-S318, 1997.

10. Tandon P and Garcia-Tsao G: Prognostic indicators in hepatocellular carcinoma: a systematic review of 72 studies. Liver Int 29 502-510, 2009

11. Di Bisceglie AM: Issues in screening and surveillance for hepatocellular carcinoma. Gastroenterology 127 (Suppl 1): S104-S107, 2004.

12. Daniele B,Bencivenga A, Megna AS and Tinessa V: $\alpha$-fetoprotein and ultrasonography screening for hepatocellular carcinoma. Gastroenterology 127 (Suppl 1): S108-S112, 2004.

13. Farinati F, Marino D, De Giorgio M, et al: Diagnostic and prognostic role of $\alpha$-fetoprotein in hepatocellular carcinoma: both or neither? Am J Gastroenterol 101: 524-532, 2006

14. Minegishi M, Tachibana K, Sato T, Iwata S, Nojima Y and Morimoto C: Structure and function of Cas-L, a $105-\mathrm{kD}$ Crk-associated substrate-related protein that is involved in $\beta 1$ integrin-mediated signaling in lymphocytes. J Exp Med 184 $1365-1375,1996$.

15. Law SF, Estojak J, Wang B, Mysliwiec T, Kruh G and Golemis EA: Human enhancer of filamentation 1 , a novel $\mathrm{p}^{3} 0^{\text {cas }}$-like docking protein, associates with focal adhesion kinase and induces pseudohyphal growth in Saccharomyces cerevisiae. Mol Cell Biol 16 3327-3337, 1996.

16. Kumar S, Tomooka $\mathrm{Y}$ and Noda M: Identification of a set of genes with developmentally down-regulated expression in the mouse brain. Biochem Biophys Res Commun 185: 1155-1161, 1992.

17. Tikhmyanova N, Little JL and Golemis EA: CAS proteins in normal and pathological cell growth control. Cell Mol Life Sci 67: 1025-1048, 2010.
18. Minn AJ, Gupta GP, Siegel PM, et al: Genes that mediate breast cancer metastasis to lung. Nature 436: 518-524, 2005.

19. Natarajan M, Stewart JE, Golemis EA, et al: HEF1 is a necessary and specific downstream effector of FAK that promotes the migration of glioblastoma cells. Oncogene 25: 1721-1732, 2006.

20. Kim M, Gans JD, Nogueira C, et al: Comparative oncogenomics identifies NEDD9 as a melanoma metastasis gene. Cell 125: 1269-1281, 2006.

21. Xue YZ, Sheng YY, Liu ZL, et al: Expression of NEDD9 in pancreatic ductal adenocarcinoma and its clinical significance. Tumour Biol 34: 895-899, 2013.

22. Guerrero MS, Parsons JT and Bouton AH: Cas and NEDD9 contribute to tumor progression through dynamic regulation of the cytoskeleton. Genes Cancer 3: 371-381, 2012.

23. Astier A, Manié SN, Law SF, et al: Association of the Cas-like molecule HEF1 with CrkL following integrin and antigen receptor signaling in human B-cells: potential relevance to neoplastic lymphohematopoietic cells. Leuk Lymphoma 28: 65-72, 1997.

24. Lucas JT Jr, Salimath BP, Slomiany MG and Rosenzweig SA: Regulation of invasive behavior by vascular endothelial growth factor is HEF1-dependent. Oncogene 29: 4449-4459, 2010.

25. Kong C, Wang C, Wang L, et al: NEDD9 is a positive regulator of epithelial-mesenchymal transition and promotes invasion in aggressive breast cancer. PLoS One 6: e22666, 2011.

26. Tikhmyanova $\mathrm{N}$ and Golemis EA: NEDD9 and BCAR1 negatively regulate E-cadherin membrane localization, and promote E-cadherin degradation. PLoS One 6: e22102, 2011.

27. Thao le B, Vu HA, Yasuda K, et al: Cas-L was over-expressed in imatinib-resistant gastrointestinal stromal tumor cells. Cancer Biol Ther 8: 683-688, 2009.

28. Nagasue N: Liver resection for hepatocellular carcinoma: indications, techniques, complications, and prognostic factors. J Hepatobiliary Pancreat Surg 5: 7-13, 1998.

29. Peng SY, Chen WJ, Lai PL, Jeng YM, Sheu JC and Hsu HC: High $\alpha$-fetoprotein level correlates with high stage, early recurrence and poor prognosis of hepatocellular carcinoma: significance of hepatitis virus infection, age, p53 and $\beta$-catenin mutations. Int J Cancer 112: 44-50, 2004.

30. Trevisani F, D'Intino PE, Morselli-Labate AM, et al: Serum $\alpha$-fetoprotein for diagnosis of hepatocellular carcinoma in patients with chronic liver disease: influence of HBsAg and anti-HCV status. J Hepatol 34: 570-575, 2001. 